\title{
EFAL: EDRS Feeder Link from Antarctic Latitudes - System Architecture and Operations Concept
}

\author{
Sergei Bobrovskyi ${ }^{*}$, Florian Sellmaier ${ }^{\dagger}$, and Felix Huber ${ }^{\ddagger}$ \\ DLR, German Space Operations Center (GSOC), Oberpfaffenhofen, Germany \\ Ricardo Barrios $^{\S}$, Dirk Giggenbach ${ }^{* *}$, and Florian Moll $^{\dagger \dagger}$ \\ DLR, Institute of Communications and Navigation (IKN), Oberpfaffenhofen, Germany
}

\section{Introduction}

The success of space born remote sensing missions depends on the quality and in most cases also on the timely availability of the recorded data. As the data quality is governed by the spatial and spectral resolution of the on-board sensors and higher resolution causes higher instrument data rates, modern sensors generate large volumes of data. The delivery of these data to the customers is however limited by the bandwidth of the radio frequency channel and by the contact times with the ground stations. The Ground Stations Networks (GSN) cannot be made arbitrarily large inter alia due to financial reasons. The topology of the chosen network introduces thereby intrinsic latency to the data distribution, which in extreme cases can be very high, e.g. if the received information has to be brought to the customer by ship. Furthermore, the amount of the information recorded per orbit on board has to be harmonized with the available downlink data rate, which therefore is driven to the one provided by the state of the art radio frequency technology, which in turn narrows down the choice of available ground stations.

The two data transfer bottlenecks: the radio frequency data rate on the one hand and the limited contact time with the ground stations on the other hand can be overcome by the utilization of optical frequencies leading to high data rates and by transferring the data via geostationary relay satellites leading to almost permanent visibility. The European Data Relay System (EDRS) embodies the optimal approach providing two payloads in the geostationary orbit (GEO) able to receive optical payload data from spacecraft in the low Earth orbit (LEO) at up to $1.8 \mathrm{Gbps}$. However, this solution may be not viable for all missions. The spacecraft in question has to be equipped with a Laser Communication Terminal (LCT) comparable with EDRS and depending on the mission characteristics, the spacecraft design, or the overall budget of the

\footnotetext{
* Research Scientist, Business Development, GSOC

${ }^{\dagger}$ Group Leader, Business Development, GSOC

‡ Director, GSOC

$\S$ Research Scientist, Advanced Optical Technologies, IKN

** Scientific Advisor, Advanced Optical Technologies, IKN

${ }^{+\dagger}$ Research Scientist, Optical Communication Systems, IKN
} 
mission, the LCT may be outside the scope of the design choices. Moreover, all already designed, manufactured or even flying spacecraft cannot be easily retrofitted with an LCT.

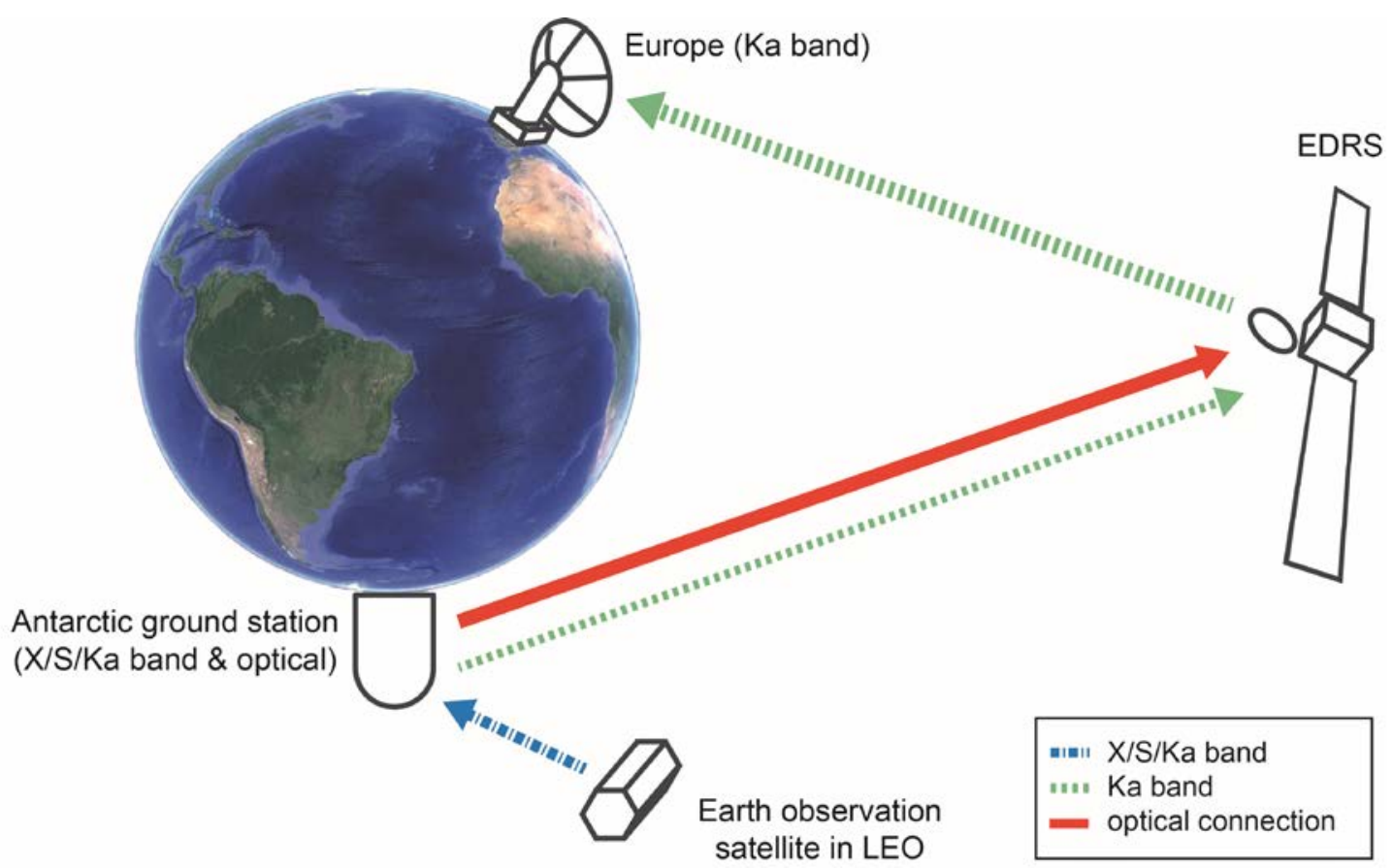

Fig. 1 Schematic view of the EFAL concept. EDRS uses several Ka band channels to downlink the data received by the optical payload.

Thus, all missions relying on conventional radio frequencies ( $X, K a$ band) for the data downlink have to optimize the GSN design in order to maximize the data throughput and to reduce the latency. As most remote sensing missions are in polar orbits to achieve full Earth coverage, long contact times to the spacecraft can be achieved from Arctic or Antarctic locations and a network encompassing ground stations in these areas allows for collection of larger amounts of data compared to other constellations. However, the sparse infrastructure in such regions usually prohibits efficient data repatriation. While Nordic stations can be connected to the existing networks via an optical cable, e.g. the Svalbard Satellite Station, this solution seems not viable for Antarctic locations. The stations there have to rely on geostationary communication satellites providing either low-bandwidth connections via global $\mathrm{C}$ band beams or solutions with higher bandwidth via expensive dedicated Ka band beams. Alternatively, the stored payload data has to be sent to the end-users by ship.

In this work we present system architecture and operations concept for an Antarctic ground station equipped with an X/S/Ka band antenna(s) for data reception employing the EDRS infrastructure for the repatriation of the user data: EDRS Feeder Link from Antarctic Latitudes (EFAL). The schematic view of the proposed setup is shown in figure 1. The use of the optical ground to space link allows for data transfer rates of up to $1.8 \mathrm{Gbps}$. In cases where the optical connection is not available, either the EDRS Ka band data relay service or the low-bandwidth bi-directional connection which is always present for the remote operations of the ground 
station can be used for the emergency data transfer depending on the maximally allowed latency. The integration of the EFAL ground station in a GSN which includes also an Arctic hub can reduce the data latency and provide the opportunity to receive telemetry and to command the user spacecraft symmetrically twice per orbit.

The major challenge of the presented scenario is the low elevation at which both EDRS spacecraft can be seen from the Antarctic coast resulting in the long atmospheric path in the lower link section imposing strong atmospheric index-of-refraction (IRT) turbulence effects onto the uplink laser beam. Additionally, the optical connection cannot be established if the laser beam is blocked by clouds. Therefore, the impact of cloud blockage and its statistics has to be taken into account in the operations concept. The preliminary results of the EFAL availability-assessment in terms of cloud blockage and the impact of atmospheric turbulence are discussed in Giggenbach [1]. The discussion includes also the preliminary evaluation of the results of an in-situ measurement campaign carried out at the Antarctic coast. The results of this study are taken into account in the present work, however, we refer the reader to Giggenbach for details [1].

This chapter is organized as follows: Section 2 describes the EFAL system architecture including the geographical constraints as well as the design of the optical ground station dependent on the challenges connected with the optical uplink through the atmosphere. The EFAL operations concept taking into account the restrictions resulting from the system design is presented in Section 3.

\section{System Architecture}

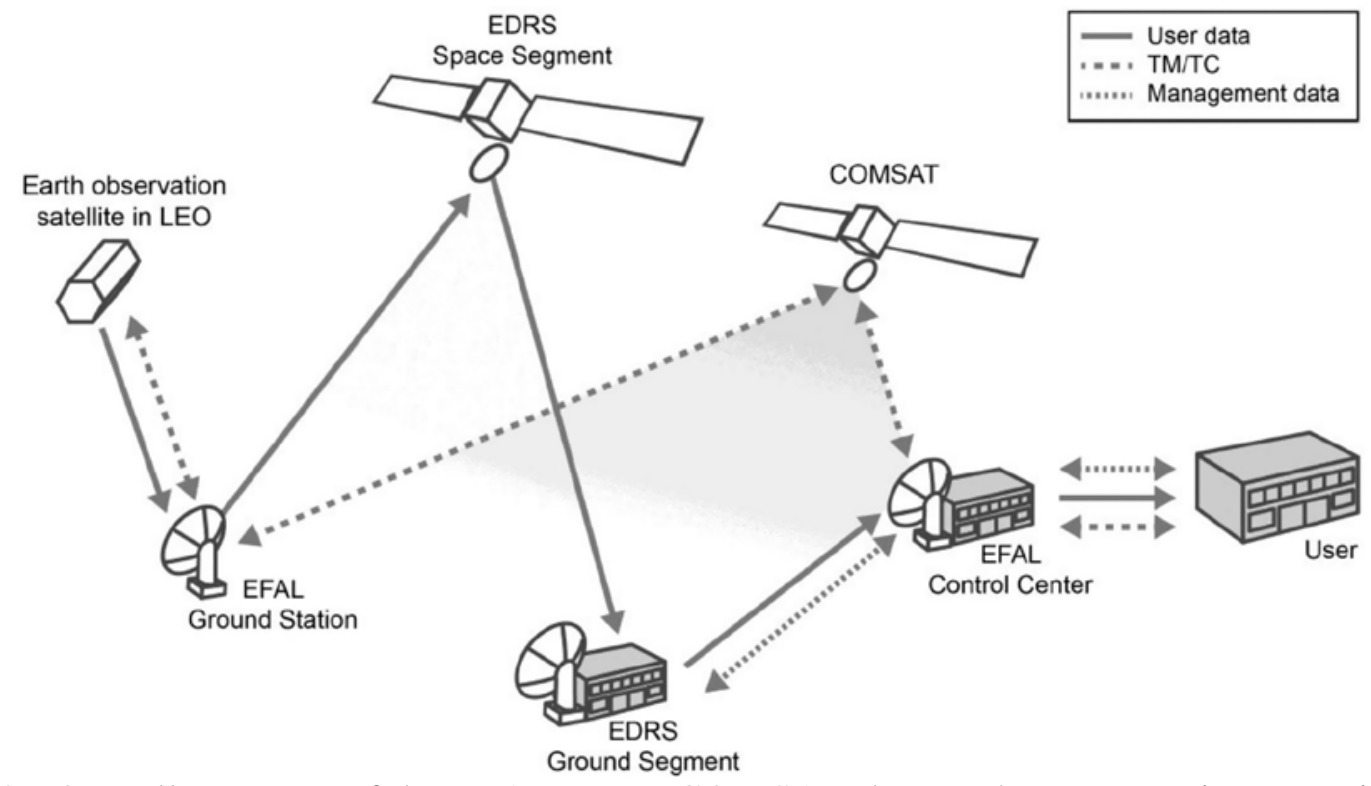

Fig. 2 Overall structure of the EFAL setup. COMSAT denotes here a generic geostationary communication satellite. TM/TC denotes here both the TM/TC data from/to user satellite as well as the data for EGS operations. 
The EFAL infrastructure consists of the EFAL Ground Station (EGS) and the EFAL Control Center (ECC) which have to be additionally connected to the EDRS Space and Ground Segments which are not official parts of the EFAL setup. The European Data Relay System is used for data repatriation and the EGS should be considered as a regular customer of the EDRS services. EGS is located in Antarctica at a site visible preferably from both EDRS spacecraft. The ECC is located in Europe and is connected to the EGS using a low-bandwidth link provided by a generic geostationary satellite. Furthermore, the ECC has a broadband connection to the EDRS Ground Segment.

The data from an Earth observation (EO) spacecraft in LEO are downlinked to the EGS which sends them then via the EDRS Space and Ground segments to the ECC which in turn delivers them then to the customer (user). The direct low-bandwidth connection between the ECC and EGS is used for the remote EGS operations. Additionally, this connection can be used for the control of the Earth observation spacecraft overflying the EGS enabling EFAL forward-tasking services in which case the user has also to provide the telecommand (TC) data. Figure 2 summarizes the overall EFAL setup.

The management data flow between the user and the ECC stands for the management activities involved in the incorporation of the EGS into the user ground network. There are also management activities concerning the interplay between the ECC and the EDRS Ground Segment, since the ECC has to request LCT communication sessions.

\section{A EFAL Ground Station}

The EFAL Ground Station consists of at least one antenna operating in the $\mathrm{X} / \mathrm{S} / \mathrm{Ka}$ band radio frequency ranges, one antenna operating in the $C$ band frequency range, a data storage facility, an optical ground station (OGS), as well as an operations system connecting the different EGS components for remote operations (EOPS). All essential components are redundant and, furthermore, interconnected and share common operational infrastructure and power generation facilities. Additionally, the EGS is equipped with a weather monitoring as well as an aviation safety system, if necessary. The EGS should be preferably maintained on-site by a small crew.

\section{Geographic Situation to EDRS Satellites}

Possible locations of the EGS can be estimated from figure 3 showing the orbital positions as well as visibility contours ( $0^{\circ}$ and $10^{\circ}$ elevation) of both EDRS spacecraft, EDRS-A being a hosted payload aboard the EUTELSAT 9B satellite. Additionally, the figure shows orbital position of the Alphasat satellite which could also be used for investigations of the optical link quality as it is equipped with a LCT for technology demonstration purposes. 


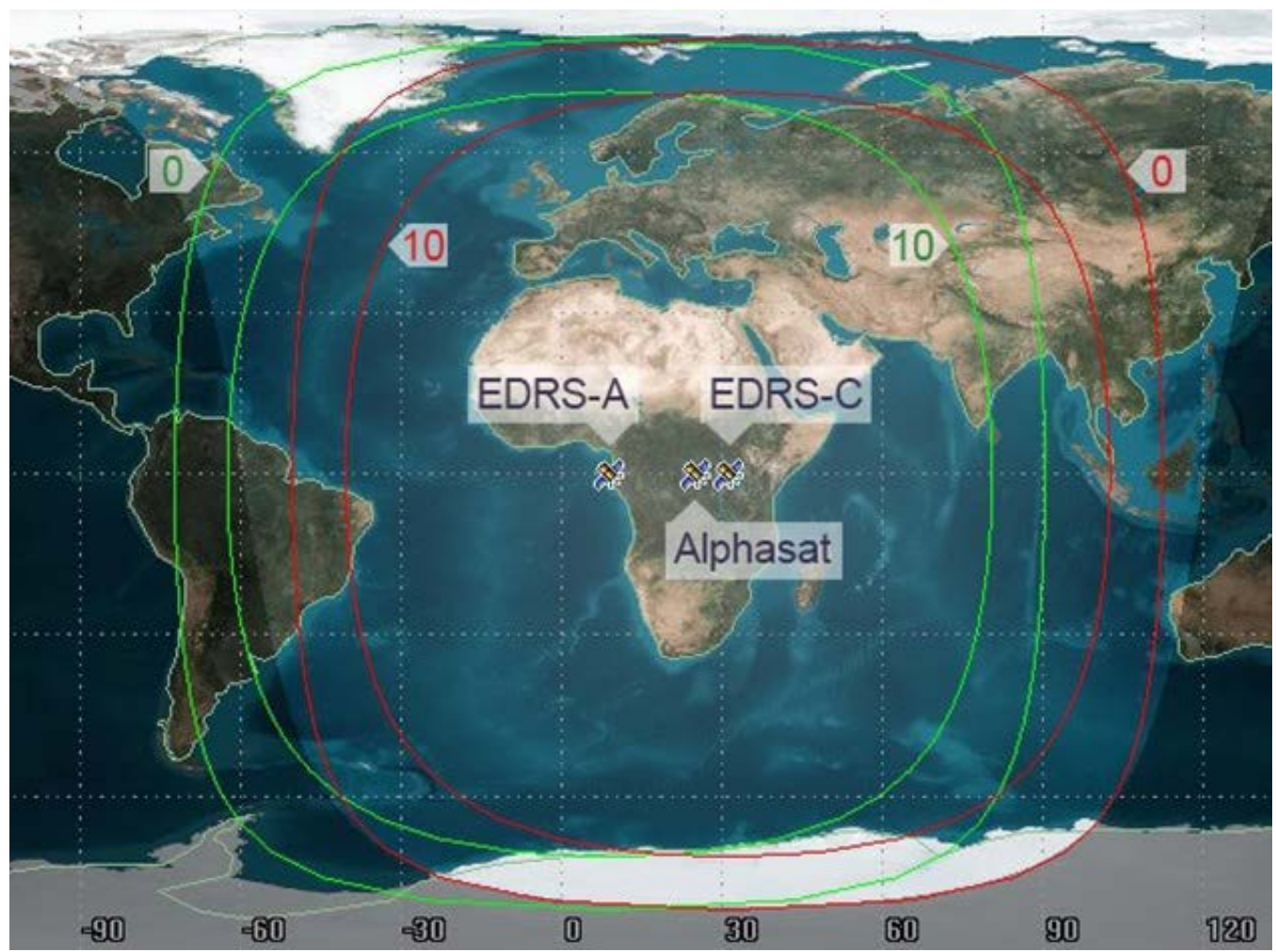

Fig. 3 Orbital positions as well as visibility contours for $0^{\circ}$ and $10^{\circ}$ elevation of the EDRS spacecraft (green EDRS-A, red EDRS-C). The orbital position of the Alphasat satellite is also shown. The Antarctic coastline east and west of $+20^{\circ}$ longitude sees theses GEO-satellites at more than $10^{\circ}$ elevation.

All aforementioned spacecraft are visible under $10^{\circ}$ elevation from a narrow strip of the Antarctic coast stretching approximately between $12^{\circ} \mathrm{E}$ and $28^{\circ} \mathrm{E}$. The range of options for a possible OGS location can be widened if the visibility of only one EDRS spacecraft is considered sufficient. As stated in the introduction, the low elevation at which both EDRS spacecraft are in general visible from the EGS poses a major challenge for the optical link and will have influence on the performance of the optical ground station needed for the data transmission.

\section{Antenna Facilities}

Table 1: Link budgets for data downlinks to the EGS.

\begin{tabular}{|c|c|c|c|}
\hline $\begin{array}{c}\text { Link Budget } \\
\text { Element }\end{array}$ & Scenario I & Scenario II & Scenario III \\
\hline X band & X band & Ka band \\
\hline Direction & downlink & downlink & downlink \\
\hline $\begin{array}{c}\text { Data rate } \\
\text { [Mbps] }\end{array}$ & 300 & 400 & 600 \\
\hline BER & $1 \times 10^{-5}$ & $1 \times 10^{-5}$ & $1 \times 10^{-5}$ \\
\hline Desired link & 6 & 6 & 6 \\
\hline
\end{tabular}


S. Bobrovskyi, F. Sellmaier, F. Huber, R. Barrios, D. Giggenbach, F. Moll, "EFAL: EDRS Feeder Link from Antarctic Latitudes System Architecture and Operations Concept", chapter 8 in "Space Operations: Innovations, Inventions, and Discoveries", series "Progress in Astronautics and Aeronautics", pp. 163-184, published by AIAA - American Institute of Aeronautics and Astronautics, November 2015. doi:10.2514/5.9781624101991.0163.0184

\begin{tabular}{|c|c|c|c|}
\hline margin [dB] & & 8.225 & 25.25 \\
\hline $\begin{array}{c}\text { Frequency } \\
{[\mathrm{GHz}]}\end{array}$ & 8.150 & 31.4 & $31.39 \dagger$ \\
\hline $\begin{array}{c}\text { Satellite EIRP } \\
{[\mathrm{dBW}]}\end{array}$ & 20 & 2770 & 2770 \\
\hline $\begin{array}{c}\text { Slant range } \\
{[\mathrm{km}]}\end{array}$ & 179.95 & 180.03 & 191.04 \\
\hline Path loss [dB] & 31.72 & 21.65 & 31.41 \\
\hline $\begin{array}{c}\text { Required GS } \\
\text { G/T [dBW/K] }\end{array}$ & 8 & 3 & 3 \\
\hline $\begin{array}{c}\text { Required GS } \\
\text { antenna } \\
\text { diameter [m] }\end{array}$ & & & \\
\hline
\end{tabular}

$\left[^{*}\right]$ Corresponds to a satellite altitude of $1000 \mathrm{~km}$ and link elevation of $10^{\circ}$. $[\dagger]$ Same antenna size as in $\mathrm{X}$ band case $(0.25 \mathrm{~m}), 32 \mathrm{dBM}$ transmitter power.

Table 2: Link budgets for data uplinks from the EGS.

\begin{tabular}{|c|c|c|}
\hline $\begin{array}{c}\text { Link Budget } \\
\text { Element }\end{array}$ & C band & Ka band \\
\hline Direction & $\begin{array}{c}\text { uplink/ } \\
\text { downlink }\end{array}$ & uplink \\
\hline $\begin{array}{c}\text { Data rate } \\
\text { [Mbps] }\end{array}$ & 1 & 300 \\
\hline BER & $1 \times 10^{-5}$ & $1 \times 10^{-5}$ \\
\hline $\begin{array}{c}\text { Desired link } \\
\text { margin [dB] }\end{array}$ & 6 & 6 \\
\hline $\begin{array}{c}\text { Frequency } \\
\text { [GHz] }\end{array}$ & $5.85 / 3.5$ & 27 \\
\hline $\begin{array}{c}\text { Slant range } \\
\text { [km] }\end{array}$ & 40655 & 40655 \\
\hline $\begin{array}{c}\text { Path loss } \\
\text { uplink [dB] }\end{array}$ & 200.32 & 214.95 \\
\hline $\begin{array}{c}\text { Required GS } \\
\text { EIRP [dBW] }\end{array}$ & 62.3 & $73.12 \dagger$ \\
\hline $\begin{array}{c}\text { Required GS } \\
\text { antenna } \\
\text { diameter [m] }\end{array}$ & $4 *$ & 3 \\
\hline
\end{tabular}

$[*]$ Assuming receiving antenna size of $4 \mathrm{~m}$.

$[\dagger]$ Estimation based on publicly accessible information.

The combined $\mathrm{X} / \mathrm{S} / \mathrm{Ka}$ band antenna is used primarily for communications with the customer EO spacecraft, since most EO spacecraft are using $\mathrm{X} / \mathrm{Ka}$ band frequency ranges for the 
data download and $\mathrm{S}$ band frequency range for the upload of telemetry and reception of the telecommands. This antenna could also be used for the repatriation of the customer data via the Ka band link service provided by EDRS-A payload in cases where the optical link is not available due to cloud blockage. It must be noted, that a dedicated Ka band uplink antenna becomes necessary, if the workload of the primary receiving antenna increases and the emergency repatriation service is considered to be critical requirement, since the primary antenna cannot fulfill both purposes at the same time. Additionally, one has also to consider the possibility to include Ku band capabilities to allow for commanding of modern spacecraft employing this frequency range for TM/TC purposes.

The primary antenna has to be a full-motion antenna able to follow the LEO-satellite. Table 1 shows link budget estimations for downlinks in $X$ and Ka bands for various spacecraft scenarios based on generic performance assumptions $[2,3]$ taking possible code gain not into account. The first scenario represents a typical EO spacecraft equipped with a small $X$ band antenna with a transmitter power in the order of $3.5 \mathrm{~W}$ [4]. An example could be TerraSAR-X [5]. The second scenario represents current and future spacecraft using state of the art X band. technologies with a dish of $0.25 \mathrm{~m}$ diameter and $7.5 \mathrm{~W}$ transmitter power [6].

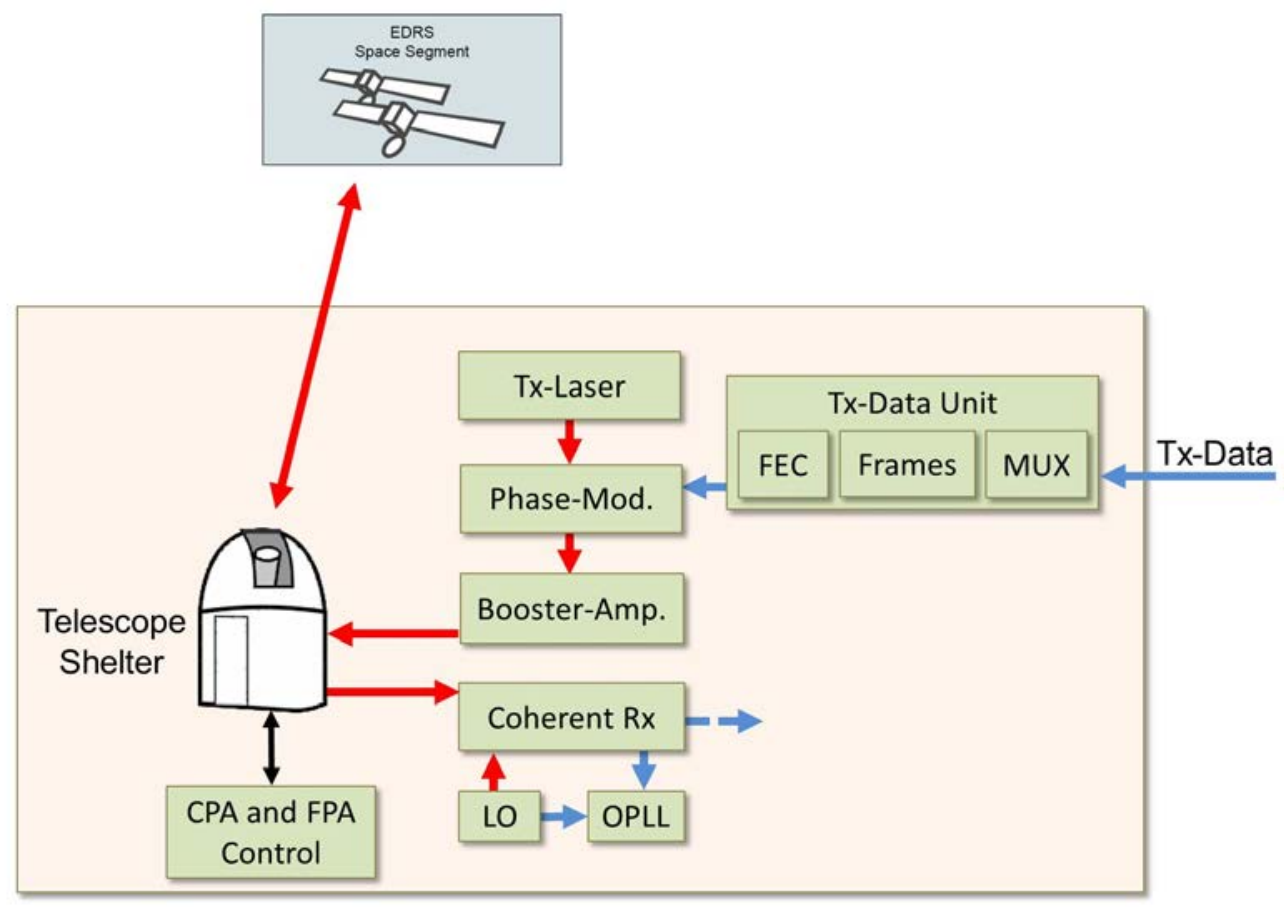

Fig. 4 Coherent optical transmission ground station structure of the EGS.

The third scenario shows requirements for data reception from a spacecraft equipped with a Ka band data downlink solution [7]. A link budget for TM/TC transmission in $\mathrm{S}$ band is not computed, since it cannot dominate the dish size due to very low required data rate. In summary, a GS antenna with a dish size of $8 \mathrm{~m}$ can receive data from most actual and future remote sensing missions.

The C band antenna is used for the remote operations of the EGS and for the 
transmission of the TM/TC streams intended for the EO spacecraft between the user and the EGS. Most geostationary communication satellites are covering both Europe and Antarctic coast with their $\mathrm{C}$ band world beams. The antenna is fixed and aimed at the geostationary satellite in question. An example for a link budget is shown in table 2 for a typical $C$ band transponder without taking code gain into account [8] [9]. A dish size of $4 \mathrm{~m}$ is required for stable data transmission assuming that the bandwidth allocated for the connection is $5 \mathrm{MHz}$ and the available power is $50 \mathrm{~W}$. The antenna on the receiving side has to have a dish size of at least 4 $\mathrm{m}$. The German Space Operations Center operates on its rooftop inter alia a $4.5 \mathrm{~m} \mathrm{C}$ band antenna able to send and receive signals [10]. It is possible to use this antenna for the transmission of signals to the EGS. However, in this case the link margin requirement has to be reduced to $3 \mathrm{~dB}$ due to limited transmitter power.

Finally, table 2 shows also the link budget for data uplink to the Ka band terminal aboard the EDRS-A spacecraft. The basis for this computation is an estimation of the transponder capability and antenna system of the Ka band terminal based on publicly accessible information and typical Ka band transponder capabilities [8] [11] [12]. The required dish size is $3 \mathrm{~m}$ assuming a transponder bandwidth of $250 \mathrm{MHz}$.

\section{Optical Ground Station}

The OGS is used for the data transmission to the EDRS space segment via a phase-modulated laser signal (Figure 4). As the optical beam divergence is in the range of few arc seconds, the OGS must be built within its own shelter on a stable foundation, to allow precise calibration of the optical axis. This will allow fast acquisitions of the link to the EDRS-GEO counter terminal, within a minimum scan time. The optical ground terminal will be positioned behind an anti-reflection coated window, operating in a controlled environment at room-temperature. The OGS consists mainly of the transceiver-telescope, steering mechanisms, acquisition- and tracking-sensors to find and track the downlink-signal from the EDRS-GEO, the laser source, optical phase modulator, the optical power amplifier, and control electronics. The Tx-Data Unit multiplexes the input data-channels, and applies framing and error protection (FEC) onto the data stream. As the standard Laser Communication Terminal establishes a bidirectional link, also the receiver part (consisting of heterodyning tracking receiver, local oscillator laser, LO, and Optical Phase-Locked Loop, OPLL) is implemented. The data-signal itself is a baseband (homodyne) BPSK signal on a $1064 \mathrm{~nm}$ wavelength optical carrier. To be able to point to both EDRS satellites plus optionally to the Alphasat position, a Coarse-Pointing Assembly (CPA) is required. The Fine Pointing Assembly (FPA) assures precise pointing and tracking of the signal direction despite IRT- or vibration-induced deviations. The required optical transmit power depends on the strength of the atmospheric IRT as the beam has to be broadened to compensate inevitable uplink beam-wander [1]. This beam-wander increases with the length of traversed IRT volume, i.e. it will be severe for a low link elevation like in EFAL.

Table 3: Link budget calculations for a PEA station to GEO uplink at $9^{\circ}$ elevation. All values in the table are given in decibels unless otherwise noted. 
S. Bobrovskyi, F. Sellmaier, F. Huber, R. Barrios, D. Giggenbach, F. Moll, "EFAL: EDRS Feeder Link from Antarctic Latitudes System Architecture and Operations Concept", chapter 8 in "Space Operations: Innovations, Inventions, and Discoveries", series "Progress in Astronautics and Aeronautics", pp. 163-184, published by AIAA - American Institute of Aeronautics and Astronautics, November 2015. doi:10.2514/5.9781624101991.0163.0184

\begin{tabular}{|l|c|}
\hline \multicolumn{1}{|c|}{ Parameter } & Value \\
\hline Tx aperture diameter [cm] & 2.10 \\
\hline Rx aperture diameter $[\mathrm{cm}]$ & 13.50 \\
\hline Fried's parameter $[\mathrm{cm}]$ & 6.10 \\
\hline Tx divergence $[\mu \mathrm{rad}]$ & 45.62 \\
\hline Beam wander $[\mu \mathrm{rad}]$ & 16.22 \\
\hline Link distance $[\mathrm{km}]$ & 40655.00 \\
\hline Tx antenna gain & 95.85 \\
\hline Tx optical loss & -3.00 \\
\hline Free-space loss & -293.63 \\
\hline Atmospheric attenuation & -2.00 \\
\hline Spreading loss & -0.81 \\
\hline Beam wander loss & -4.39 \\
\hline Rx antenna gain & 112.01 \\
\hline Rx optical loss & -3.00 \\
\hline Total link loss & -99.00 \\
\hline Additional link margin & -2.00 \\
\hline Rx Sensitivity BER=10E-8 & -51.00 \\
\hline Required Tx power $[\mathrm{dBm}]$ & 50.00 \\
\hline Required Tx power $[\mathrm{W}]$ & 99.92 \\
\hline
\end{tabular}

Therefore, required transmit powers of several tens of watts are being expected. Use of such a powerful laser in the open field requires paying attention to eye-safety issues like aviation safety. However, these can be controlled by established techniques like radar-sensors. Interference with air-traffic will be extremely low at the Antarctic location, as the beam will have reached an eye-save diameter at the usual aircraft cruising altitudes. Since a laser link through the atmosphere can only be established if there is a cloud free line of sight between the transmitter and the receiver, a cloud monitoring facility is essential for OGS operations.

For the optical link budget calculation, using the in-situ $r_{0}$ value, characterizing the optical turbulence, estimated by a measurement campaign [1], all the pertinent parameters and calculations are presented in Table 3, where parameter values defining the link scenario are in the upper part of the table, and the values included in the final link budget calculations are shown in bottom part of the table. Here it can be seen that requiring a data rate of $600 \mathrm{Mbps}$ the link is possible for the median value of the Fried's parameter $\left(r_{0}=6.1 \mathrm{~cm}\right)$ measured at the Princess Elisabeth Antarctica (PEA) research station [1], as the required power of $99 \mathrm{~W}$ can be reached with an optical amplifier working at a wavelength of $1 \mu \mathrm{m}$ [13]. Please note that the removal of the additional link margin $(2 \mathrm{~dB})$ reduces the required Tx-power to about $60 \mathrm{~W}$.

\section{Storage Facility}


It cannot be guaranteed that all user data received during one downlink can be simultaneously transmitted to the customer and therefore a storage facility is a mandatory element of the EGS. The storage capacity has to be chosen such, that on the one hand the customer data accumulated during some very long outage is not lost and other other hand all data stored during such outage can be send to the users before the next long outage occurs, because otherwise more and more data will pile up. The actual design depends on the details of the cloud cover analysis, see Section 3.1, which in turn influences the availability of the station. In general, the duration of the link fade due to cloud cover will have a mean value $\tau_{\text {Fade }}$. However, the probability of much longer fades is not zero and an appropriate measure has to be chosen to select the longest fade $t_{\mathrm{Fade}}^{\max }$ during which all data has to be stored and can be stored, called the longest tolerable fade in the following. For fades longer than $t_{\text {Fade }}^{\max }$ only the part of the data which can be stored during $t_{\text {Fade }}^{\max }$ is stored and the rest will be lost. The selection of $t_{\text {Fade }}^{\max }$ should be such, that all the data stored during the last long outage can be transmitted to the customers before the next long outage occurs, because otherwise the data will indefinitely accumulate in the storage. A necessary condition for the previous statement is that the amount of data which can be transmitted during a typical link with duration $p_{\text {eff }} \cdot \tau_{\text {Link }}, \tau_{\text {Link }}$ being the mean link duration and $p_{\text {eff }}$ the fraction of link time available for contact, is larger than amount of data downlinked from the customer satellites during the previous fade as well as the full cloud free time $\tau_{\text {Fade }}+\tau_{\text {Link }}$. Thus, during one typical link-fade cycle some extra amount of data $\Delta d$ can be transmitted to the customers in addition to the data received during the cycle itself. If $n$ typical link-fade cycles occur between the occurrences of the longest tolerable fade, then the total amount of data which in principle can be send additionally away $n \cdot \Delta d$ should be larger or at least equal to the total amount of data stored during the last long fade $t_{\text {Fade }}^{\max } \cdot \rho_{\text {down }}, \rho_{\text {down }}$ being the mean data rate of customer downlinks. If the newest data is always sent to the customers first, then the maximal latency of the stored data in the case $n \cdot \Delta d=t_{\text {Fade }}^{\text {max }} \cdot \rho_{\text {down }}$ is exactly the time between successive longest tolerable fades. Since in some cases the latency of the data has to be minimized, c.f. Section 3.2, some part of the data received during the long fade will be sent to customers via the Ka band uplink to EDRS-A leading to a lower effective downlink datarate during the fade $\left(\rho_{\mathrm{down}}-p \cdot r_{\mathrm{up}}^{\mathrm{Ka}}\right), r_{\mathrm{up}}^{\mathrm{Ka}}$ being the Ka band uplink data rate and $p$ fractional amount of time in units of the fade time during which the uplink takes place. Summing up, after the determination of $t_{\mathrm{Fade}}^{\max }$, the storage device can be designed to have the minimum capacity of $\left(\rho_{\text {down }}-p \cdot r_{\text {up }}^{\mathrm{Ka}}\right) \cdot\left(t_{\text {Fade }}^{\text {max }}+\tau_{\text {Fade }}\right)+\rho_{\text {down }} \cdot \tau_{\text {Link }}$, which means that it should at least have the capacity to store the data received during the last long fade and one typical link-fade cycle. An example for the estimation of the storage device size is shown in Appendix A.

\section{B EFAL Control Center}


S. Bobrovskyi, F. Sellmaier, F. Huber, R. Barrios, D. Giggenbach, F. Moll, "EFAL: EDRS Feeder Link from Antarctic Latitudes System Architecture and Operations Concept", chapter 8 in "Space Operations: Innovations, Inventions, and Discoveries", series "Progress in Astronautics and Aeronautics", pp. 163-184, published by AIAA - American Institute of Aeronautics and Astronautics, November 2015. doi:10.2514/5.9781624101991.0163.0184

The EFAL Control Center consists of a $\mathrm{C}$ band antenna, a weather forecast system coupled to a system operating the optical ground station (OGS-OPS), a system operating remotely the antennas of the EGS and the data storage at the EGS (DATA-OPS), as well as a management facility responsible for the interface to the EDRS Ground Segment and to the users.

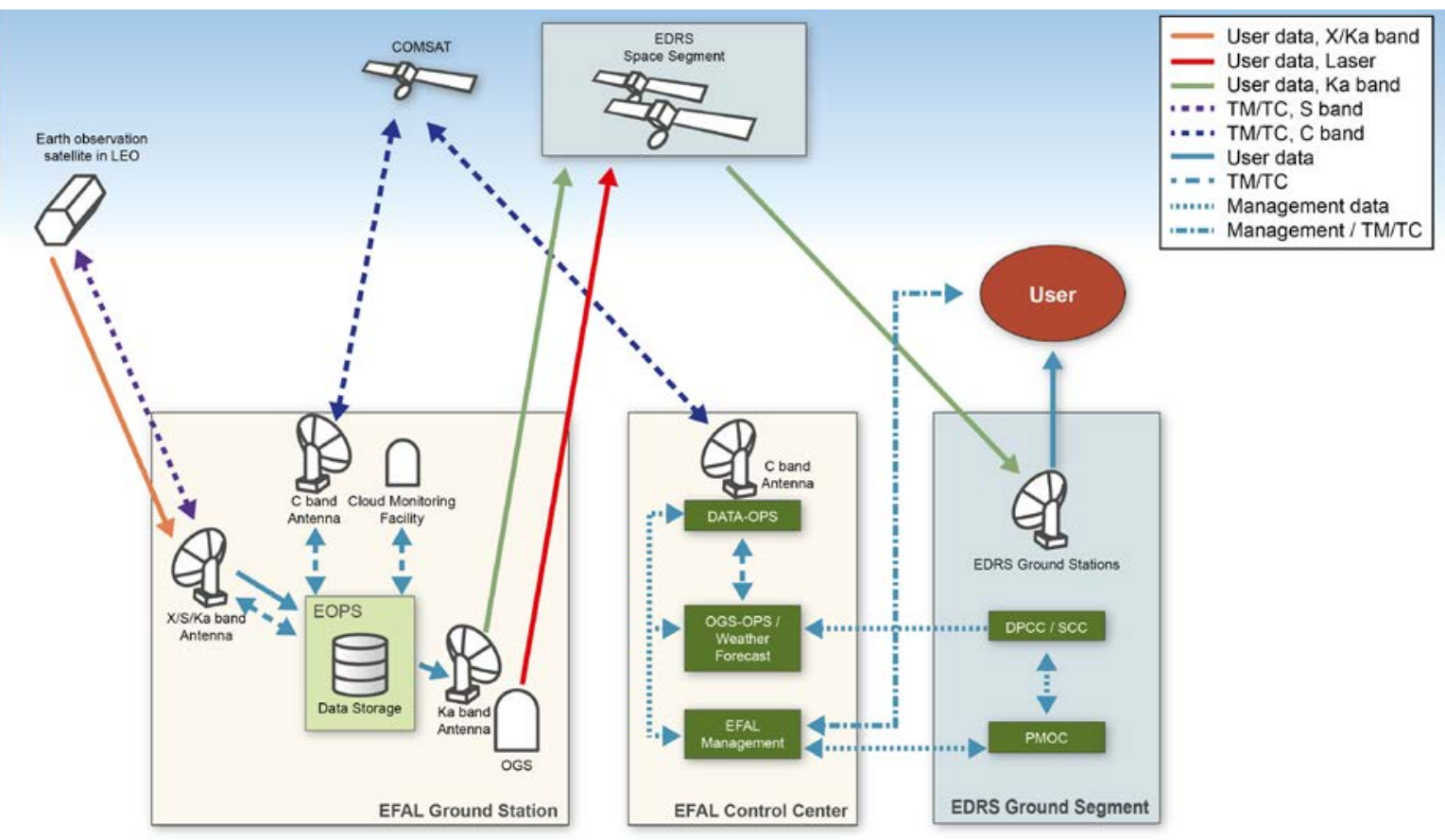

Fig. 5 Possible implementation of the EFAL system architecture. This figure shows only those parts of the data flow inside the EDRS ground segment which are of the importance for the understanding of the EFAL project. TM/TC denotes here both the TM/TC data to/from user satellite as well as data needed for the EGS operations.

This management facility is also connected to the system operating the OGS, in order to coordinate the transmission of the user data via the OGS and the pointing of the Laser Communication Terminal aboard one of the EDRS spacecraft used for the connection. The $C$ band antenna is used for the transmission of TM/TC data to and from the user spacecraft, the transmission of commands to the EGS, and the transmission of the weather data from the EGS to the ECC.

In the following we consider a possible implementation of the EFAL Control Center at the German Space Operations Center (GSOC) in Oberpfaffenhofen, which is responsible for the operations of both EDRS payloads in the Devolved Payload Control Center (DPCC) and the Satellite Control Center (SCC) the latter being also responsible for the operations of the EDRS-C satellite, as well as central EDRS ground stations [14]. The operations of the overall European Data Relay Systems are performed by Airbus Defence and Space within the EDRS Primary Mission and Operations Center (PMOC). The establishment of the ECC at GSOC can simplify the operational aspects of the EFAL setup due to the access to the EDRS LCT and Ka band pointing schedule. Moreover, the data transmitted via EDRS can be delivered to customers directly from 
the EDRS ground stations. However, it must be noted that any other implementation which can have in principle an access to the schedule (it is enough to know which EDRS contact slots are not available) and has a broadband connection to the EDRS ground segment for user data delivery is equally adequate. Figure 5 shows a possible implementation of the full EFAL architecture.

\section{Operations Concept}

The EFAL setup corresponds to an Antarctic ground station. A user can book passes over the EGS and will receive the data collected by the EO spacecraft some time later from the European location. The latency between the data downlink and reception in Europe depends on the cloud cover over the EGS and therefore a particular latency can be guaranteed only if the Ka band uplink to EDRS-A is also used for data repatriation.

The operations concept can be divided in two parts, one part concerning the operations of the radio frequency antennas and all EGS facilities except for the OGS, and OGS operations determined by the scenario of data repatriation.

The regular ground station operations can be summarized in following points:

- Customers (users) are booking passes over the EGS and provide detailed information on the orbit of the EO satellite to the EFAL management. Users are responsible for the start of the data downlink, correct pointing of the EO spacecraft, and provision of TC sets in cases where the spacecraft should receive new commands,

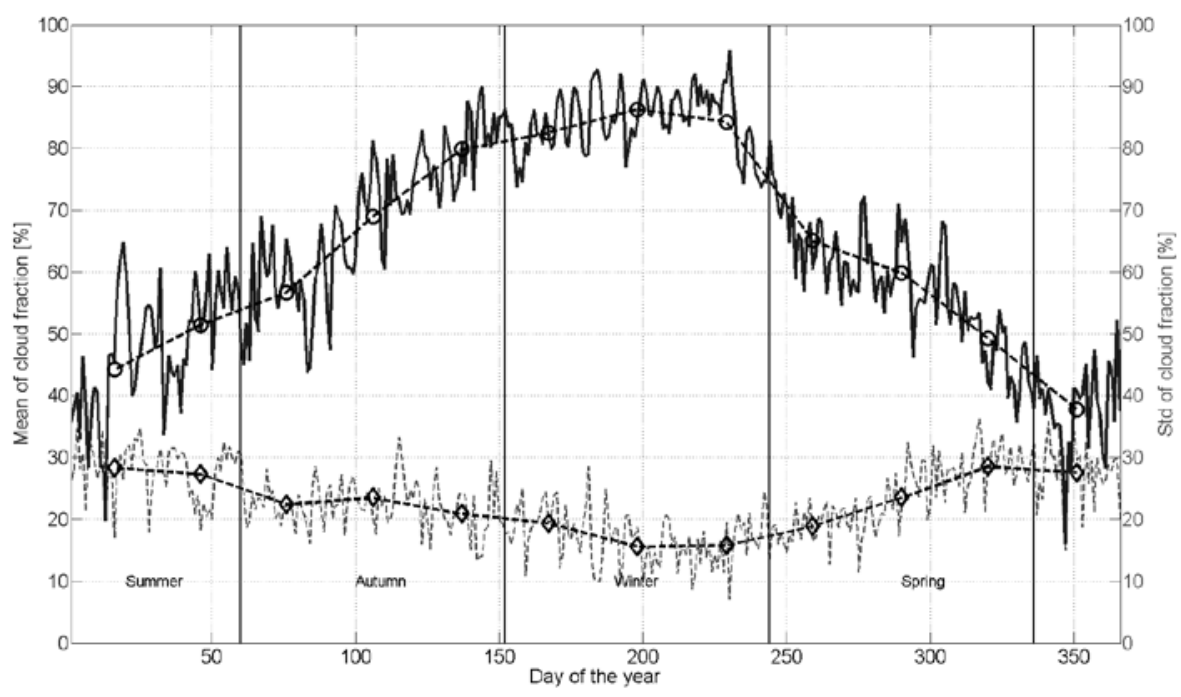




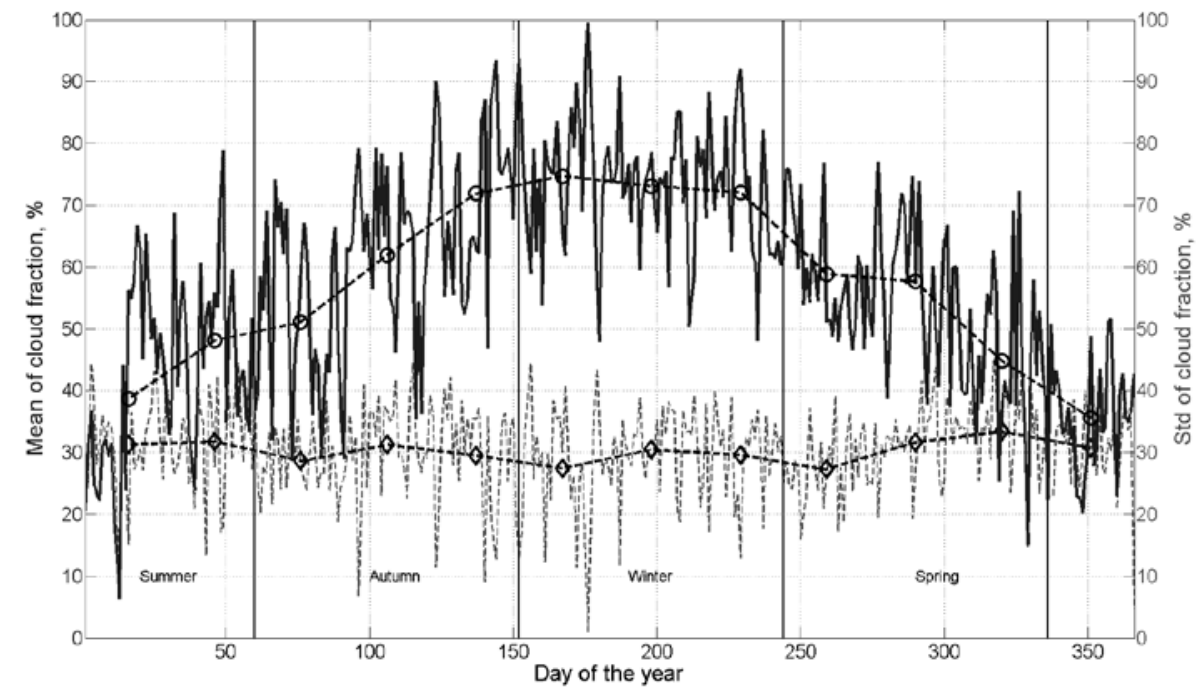

Fig. 6 Course of the year of cloud cover over PEA. The values are based on images from the Terra satellite (top) (years 2000 to 2013) and the Aqua satellite (bottom) (2002 to 2008). The blue line denotes mean cloud fraction for the day of the year, the black dashed line with circular markers the according monthly averages. The dashed green line is the related standard deviation and the black dashed line with diamond markers denotes the according monthly averages.

- DATA-OPS is remotely operating the X/S/Ka band antenna of the EGS monitoring also the state of the mass storage and making sure that the user data is received an correctly stored. It is also responsible for the transmission of TM/TC data between the satellite and the user.

Concerning the data repatriation from the Antarctica one can envisage two scenarios:

- Low latency scenario The aim of this scenario is to bring the downlinked data as fast as possible to the customer. Preferably, the laser link is being used, however, the Ka band uplink to EDRS is used as fast as possible if the laser link is not available due to clouds.

- Background scenario The aim of this scenario is to transfer large amounts of data from the Antarctica to Europe via optical laser link without interfering with the usual operations of the LCTs aboard the EDRS spacecraft.

The rationale for this two scenarios could be a different pricing schemes for each case.

\section{A. Cloud Cover Statistics}

Clouds in the line of sight most often prohibit the optical link due to exorbitantly high attenuation. This can be as high as 100 up to $600 \mathrm{~dB} / \mathrm{km}$ for water cloud types like Cumulus, Stratus, Stratocumulus, Altostratus, and Nimbostratus, and even the thinnest Stratus and Stratocumulus clouds exceed over $30 \mathrm{~dB}$ of total attenuation [15]. Ice clouds have lower 
attenuation due to lower mass density than water clouds, bearing values of 1-6 dB/ $\mathrm{km}$ and 1-15 $\mathrm{dB}$ total attenuation [16]. Eventually, only very thin water clouds and ice clouds have attenuation that is low enough to be reasonably regarded in a link budget. But in most cases the link is inhibited. This causes the need to statistically analyze cloud occultation over the site of interest. In the present work, we use MODIS data for that purpose due to the large available data base, easy access to the necessary data products, reasonable spatial resolution $\left(1^{\circ}\right.$ grid map resolution) and good visibility from the PEA Station This station serves as a representative of a possible EGS location and is the site where our in-situ atmospheric measurement campaign has taken place [1]. The MODIS Level 3 Atmosphere Products MOD08 [17, 18] are chosen and from these the parameter Mean Cloud Fraction. The mean cloud cover at PEA over the year derived from the data acquired by MODIS instrument aboard the Terra and Aqua satellites is shown in figure 6. The values are based on images from the Terra satellite (years 2000 to 2013) and the Aqua satellite (2002 to 2008). A difference in the course of the graphs is clearly visilble. The Terra (morning) images reveal higher cloud fraction than the Aqua (afternoon) images. The mean decrease is around $5 \%$.

This measurement just shows the measured mean cloud fraction, i.e. the mean fractional fade time over the year. As one can see, this fade time can be as high as $90 \%$ during the Antarctic winter, which however would still mean that three full days without clouds are available for optical data uplink. Also the difference between the Terra and Aqua data suggest a somewhat lower average cloud cover value.
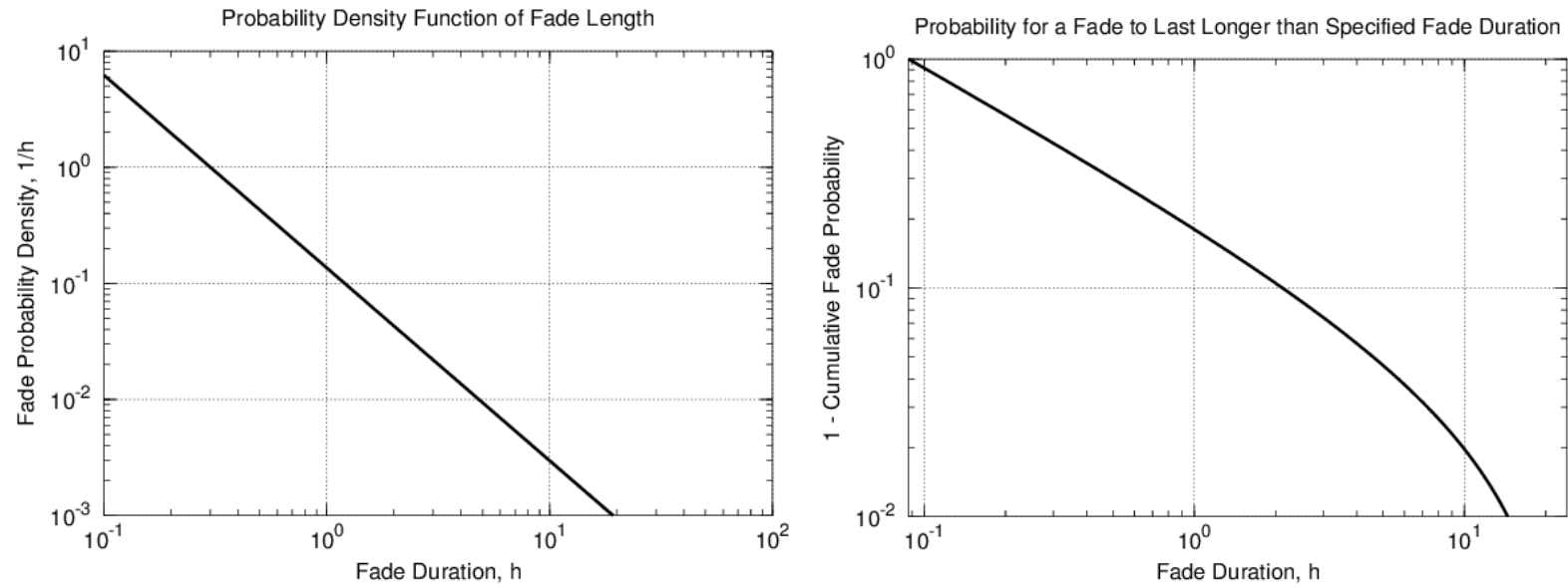

Figure 7: Example for the probability density function of the fade duration (left) and the probability for a fade to last longer than given fade duration (right) for a cloud statistics satisfying $\tau_{\text {Fade }}=1 \mathrm{~h}$.

Nevertheless, it is important to know how the cloud-free days are distributed throughout a full month. Therefore, the temporal evolution of the blockage is also of high importance to estimate link and outage times for operations. In Perlot [19], a modelling approach for availability and unavailability of single site links and networks is presented. Here, a lognormal model is arbitrarily chosen to assess the dependency of link and network availability on fade and link 
duration. Analysis of data from aircraft observations, satellite measurements (MODIS) and model predictions show that cloud chord length $l$ measured in meters usually follows a single exponential behavior[20]:

$$
f(l)=\alpha \cdot l^{-\beta},
$$

where the numerical coefficient $\beta$ is found to be 1.66 . This equation is only valid for a range of cloud sizes. Applying a particular wind speed $v$ measured in $\mathrm{m} / \mathrm{s}$, the probability density distribution of link outage times can be estimated. This would lead to

$$
p^{\text {Out }}(t)=\alpha(v t)^{-\beta}
$$

where $t$ is a duration of link outage. Note that also in this case a minimal and a maximal fade durations which depend on the cloud sizes corresponding to the validity region of Eq. 1 and wind speeds in question have to be assumed. As one can see, the frequency of fades monotonously decreases with fade duration. However, the actual shape of the fade duration probability density function (PDF) or cloud size PDF at PEA is presently not known. Therefore, throughout this work we will use the abstract quantities mean fade duration $\tau_{\text {Fade }}=<t>$, where the expectation value is taken with respect to the PDF in Eq. 2 and mean link duration $\tau_{\text {Link }}$, which is defined similarly to $\tau_{\text {Fade }}$ with the PDF of link duration. In numerical examples we will simply and arbitrarily set the mean fade duration $\tau_{\text {Fade }}$ to $1 \mathrm{~h}$ and mean link duration $\tau_{\text {Link }}$ to $0.88 \mathrm{~h}$ implying slightly different probability distributions for link and fade lengths. Figure 7 illustrates the form of the PDF for a cloud cover statistics with $\tau_{\text {Fade }}=1 \mathrm{~h}$ assuming that the model is valid for fade durations up to $24 \mathrm{~h}$. The figure shows also the associated probability that a fade lasts longer than the specified fade duration. As one can see, most fades are very short, as expected, the probability for a fade to be longer than $1 \mathrm{~h}$ being $18 \%$. For operational concepts one also needs the correlation time of cloud occurrence $\tau_{\text {corr }}$, which has to be derived from the true cloud statistics.

\section{B. Low latency Scenario}

This scenario is defined by the requirement that the data is sent to the users as fast as possible after the reception. We assume that the service of the EDRS system can be booked maximally one hour before the envisaged connection. Therefore, the weather forecast system of the ECC should predict the weather conditions at the EGS using the EGS cloud monitoring facility as well as other sources one hour before the envisaged end of the data downlink from the customer satellite. If the weather forecast system is sufficiently complex, its output is a probability value $p_{\text {Cloud }}$ that the sky is either cloudy at the envisaged beginning of the link or will become cloudy during the link. The actual threshold value for this probability must be determined in an optimization process. For the purpose of this work we will arbitrarily assume a value of 0.02 corresponding to 28.8 cases of link blockage due to clouds per months in situations where no 
clouds were expected. Additionally, the full system has to take into account the reliability of the weather forecast system itself.

If $p_{\text {Cloud }} \geq 0.02$ a Ka band link to EDRS-A is requested from PMOC by EFAL management. In other case, an LCT link to either one of the EDRS spacecraft is requested from PMOC. It is important to note, that in case of many short fades between somewhat longer clear sky times, as it is indicated by the cloud cover statistics in Section 3.1, an intelligent filter has to be employed to smooth over the fade times, since a connection which is interrupted for a short time but can be continued and brought to an end is still a viable option.

Should a scheduled LCT connection become impossible due to clouds contrary to the weather prediction, the weather forecast and scheduling procedure is repeated again with updated weather information and tightened good-weather condition, e.g. Ka band link if $p_{\text {Cloud }} \geq 0.01$, taking into account now longer required uplink time, which in turn can have impact on the outcome of the probability calculation.

The operations of the OGS are performed remotely from the EFAL Control Center by OGS-OPS system.

Even without a sophisticated weather prediction system, but knowing the cloud cover statistics, in particular the correlation time of cloud occurrence $\tau_{\text {Corr }}$, for preferably each Antarctic season of year, one can envisage a solution for the low latency operations scenario. One hour before the envisaged start of the transmission one has to check for actual cloud cover:

- If the sky is clear and $\tau_{\text {Corr }}>\left(\Delta t_{\text {avail }}+\Delta t_{\text {Upload }}\right)$, where $\Delta t_{\text {avail }}$ is the time between the current time and the envisaged start of transmission which is $1 \mathrm{~h}$ if the LCTs are always available, and $\Delta t_{\text {Upload }}$ is the predicted duration of data transmission, then laser connection is requested from PMOC. If the inequality is not satisfied, a Ka band connection is requested from PMOC. Additionally, one could check whether a cloud cover or other dramatic weather change is predicted for the next hour from other weather measurement devices at the EGS and change the requests adequately.

- If the sky is cloudy and $\tau_{\text {Corr }}<1 \mathrm{~h}$ then an LCT connection is requested from PMOC. Here, we assume that $p_{\text {eff }} \cdot \tau_{\text {Link }}$, which is the effective available link time taking on average into account the unavailability of the LCTs due to requests of other customers during cloud free time at the EGS, is always larger than $\Delta t_{\mathrm{Upload}}$. In the other case, a Ka band connection is requested from PMOC.

Since the ECC can have access to the EDRS LCT and Ka band terminal schedules, the link requests can be scheduled such that they start as fast as possible after each downlink without interfering with existing requests from other customers.

\section{Background Scenario}

The aim of the background scenario is to use only the optical connection to EDRS while 
minimizing the number of required connections. This aim is translated into requirement to save the downloaded data in the storage system until the amount of saved data equals the amount of data which can be uploaded during the longest available LCT time slot, which is bounded on average by $\tau_{\text {Link }}$. Saving more data would lead to accumulation of data in the storage. Since the available time is bounded by $\tau_{\text {Link }}$, it is possible to estimate the longest duration during which all the downloaded data can be stored:

$$
t_{\text {max }}=\tau_{\text {Link }}\left(\frac{r_{\mathrm{up}}^{\mathrm{LCT}}}{\rho_{\mathrm{eff}}}-1\right),
$$

where $\rho_{\text {eff }}$ is the effective downlink data rate which takes into account, that some user data is regularly uploaded either via Ka band or laser link due to low latency requirement. The effective downlink datarate $\rho_{\text {eff }}$ is equal to the quantity $\left(\rho_{\text {down }}-p \cdot r_{\text {up }}^{\mathrm{Ka}}\right)$ introduced in Section 2.1.4 assuming that the same amount of data is immediately send to the customers due to low latency requirements irrespective of the used channel (Ka band or laser link). Assuming the values from Appendix A and $\rho_{\text {eff }}=300 \mathrm{Gbit} / \mathrm{h}, t_{\max }=5.45 \mathrm{~h}$. The operations for the background scenario begin right after the last successful uplink.

- Weather predictions and LCT availability are correlated for the next $t_{\max }$. The LCT schedules are assumed to be known as in the low latency scenario. Additionally, LCT requests from low latency operations are also taken into account and have priority. The result of this correlation are all time slots during which a connection to the EDRS LCTs can in principle take place as well as the starting times of these time slots.

- In the next step the longest consistent time slot available for uplink $\tau_{\text {avail }}^{L C T}$ is being determined. Its duration is characterized by the condition that this is the longest time slot starting before or right at its own optimal start time $t_{\mathrm{opt}}$ given by:

$$
t_{\mathrm{opt}, i}=\tau_{i}^{\mathrm{LCT}}\left(\frac{r_{\mathrm{up}}^{\mathrm{LCT}}}{\rho_{\mathrm{eff}}}-1\right)-d_{\text {Residue }},
$$

where $d_{\text {Residue }}$ is the residual content of the storage at the time of the prediction. The optimal start time is a theoretical value different for each available time slot $\tau_{i}^{\text {LCT }}$. Their true starting times are compared with this value and then $\tau_{\text {avail }}^{L C T}$ is chosen according to the condition stated above.

- At the time $t_{\text {Link }}-1 \mathrm{~h}, t_{\text {Link }}$ being the previously found star time of the optimal time slot which is equal to $t_{\mathrm{opt}}$ in the best case, LCT schedule and weather conditions are checked again. If LCT link seems to be possible it is requested from PMOC. Otherwise the procedure starts again with updated value of $d_{\text {Residue }}$.

One has to note, that at some point $t_{\mathrm{opt}}$ can become less than one hour or even negative in 
which case it is set to one hour, meaning that the program starts to use all available time slots for data transmission until that time rises again. In the extreme case of the scenario presented in Appendix A, where fades of 48 hours occur every $55 \mathrm{~h}$, all available LCT time slots are being used for data uplinks between the long fades. The data coming from low latency customers would still have priority. Figure 8 shows a schematic representation of the background data repatriation scenario.

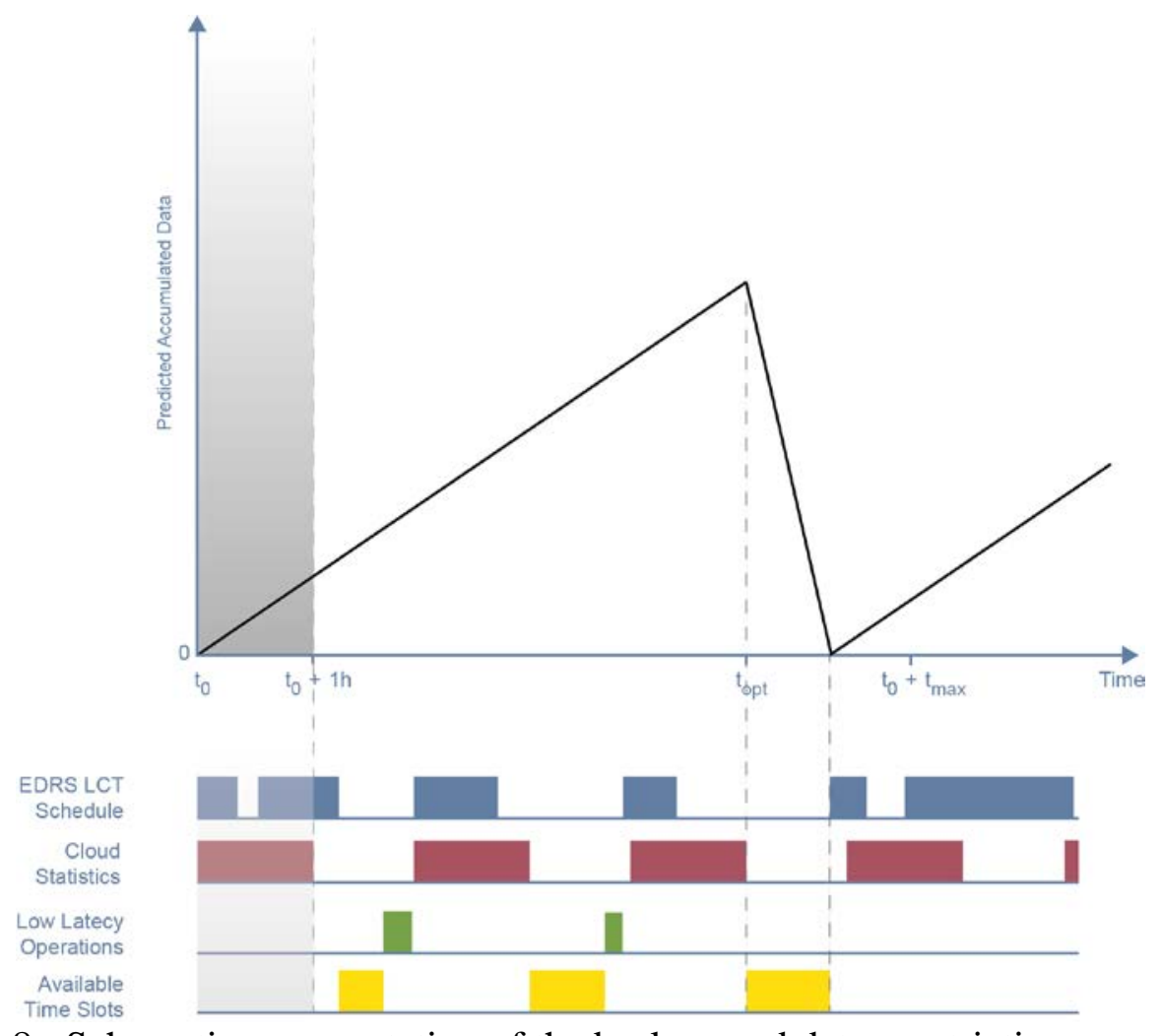

Fig. 8 Schematic representation of the background data repatriation scenario.

The graph shows the predicted amount of data in storage as a function of time after the last upload. No uplinks can be scheduled in the shaded area. The lower part shows correlations between cloud cover, $\tau_{\text {Fade }}$ being shown in red and $\tau_{\text {Link }}$ being the time in between, and EDRS LCT availability due to external customers in blue and EFAL low latency operations in green. Yellow bands represent all available time slots for background uplinks, $\tau_{\text {avail }}^{L C T}$ being the last yellow band.

\section{Appendix A}

This appendix shows an example for a possible determination of the storage device size and clarifies the notation employed in Section 2.1.4. We assume that we know $\tau_{\text {Link }}$ and $\tau_{\text {Fade }}$ from cloud cover analysis at the site of the EGS and set them to $\tau_{\text {Link }}=0.88 \mathrm{~h}$ and $\tau_{\text {Fade }}=1 \mathrm{~h}$. We take the mean downlink data rate to be $\rho_{\text {down }}=600 \mathrm{Gbit} / \mathrm{h}$, which corresponds to two 
downlinks per hour at $500 \mathrm{Mbit} / \mathrm{s}$, each with a duration of 10 minutes. We furthermore set $r_{\text {up }}^{\mathrm{LCT}}=2160 \mathrm{Gbit} / \mathrm{h}$ corresponding to a data rate of $600 \mathrm{Mbit} / \mathrm{s}$ and $r_{\mathrm{up}}^{\mathrm{Ka}}=1080 \mathrm{Gbit} / \mathrm{h}$ corresponding respectively to a data rate of $300 \mathrm{Mbit} / \mathrm{s}$. Moreover, we set $p=0.28$ and $p_{\text {eff }}=0.7$, which means that approximately half of the data downloaded during the fades is immediately send to the customer via Ka band and that the LCTs aboard the EDRS spacecraft are available for EFAL uplink on average $70 \%$ of the mean link time. It follows:

$$
\Delta d=\left(p_{\text {eff }} \cdot r_{\text {up }}^{\text {LCT }}-\rho_{\text {down }}\right) \tau_{\text {Link }}-\left(\rho_{\text {down }}-p \cdot r_{\text {up }}^{\text {Ka }}\right) \tau_{\text {Fade }}=504.96 \mathrm{Gbit} .
$$

At this stage one would need times between two successive long fades for each different long fade duration. Let us assume that we want the longest tolerable fade to be $t_{\mathrm{Fade}}^{\max }=48 \mathrm{~h}$. In this case, we have:

$$
\left.n=\left(\rho_{\text {down }}-p \cdot r_{\text {up }}^{\text {Ka }}\right)\right)_{\text {Fade }}^{\max } / \Delta d \cong 29
$$

which means that fades of $48 \mathrm{~h}$ can occur at most every $29 \cdot\left(\tau_{\text {Link }}+\tau_{\text {Fade }}\right)=55 \mathrm{~h}$ and that all data downlinked during such fade will be received by the customer at most 55 hours after the fade. However, one has to keep in mind that we have assumed that approximately half of the downlinked data is transferred to customer without delays via the Ka band connection. The minimal size of the needed storage device including some margin can be estimated to be

$$
\left(\rho_{\text {down }}-p \cdot r_{\text {up }}^{\text {Ka }}\right)\left(t_{\text {Fade }}^{\max }+\tau_{\text {Fade }}\right)+\rho_{\text {down }} \tau_{\text {Link }} \cong 2 \text { Tbyte. }
$$

Finally, we have to point out, that in this extreme case the laser links have to take place during each available link time ruling out the possibility of the background service.

\section{References}

[1] Giggenbach, D., Barrios, R., Moll, F., Calvo, R. M., Bobrovskyi, S., Huber, F., Johnson-Amin, N. F., Heine, F., and Gregory, M., "EFAL: EDRS Feeder Link from Antarctic Latitudes - Preliminary Results of Site Investigations, Availability, and System Requirements,” ICSOS 2014, International Conference on Space Optical Systems and Applications, Kobe, Japan, May 2014.

[2] Maral, G. and Bousquet, M., Satellite Communications Systems, John Wiley \& Sons Ltd, New York, 2009.

[3] Dodel, H. and Eberle, S., Satellitenkommunikation, Springer, Berlin, 2007.

[4] Tesat Spacecom, "Modulators and Transmitters," July 2012, http://www.tesat.de/en/divisions/datalink-products/modulators-and-transmitters, [Last Accessed on March 23, 2014].

[5] Werninghaus, R. and Buckreuss, S., "The TerraSAR-X Mission and System Design,” IEEE Transactions on Geoscience and Remote Sensing, Vol. 48, 2010, pp. 606-614.

[6] Hartzell, C. M., Graham, L. C., Tao, T. S., R.Goldberg, H., Carpena-Nunez, J., Racek, D. 
M., Taylor, C. E., and Norton, C. D., “Data System Design for a Hyperspectral Imaging Mission Concept,” 2009 IEEE Aerospace Conference, Big Sky Montana, March 2009.

[7] Space Micro, “ $\mu$ Tx-300 Ka-Band Transmitter,” June 2012, http://www.spacemicro.com/assets/ka-band-v7.0.pdf, [Last accessed on March 17, 2014].

[8] Eberle, S. and Wörfel, R., "Satellitenkommunikation: Orbits, Frequenzen, Verfahren, Systeme, SATCOMBw, UN-Missionen,” Febuary 2012.

[9] SES, “SES Fleet and Coverage,” August 2011, http://www.ses.com/19487010/ fleet-and-coverage, [Last accessed on September 3, 2013].

[10] Fries, R., "Space Operations and Astronaut Training - Communications and Ground Stations," Space Operations and Astronaut Training Seminar, Oberpfaffenhofen, Germany, September 2013.

[11] ESA, “European Data Relay Satellite System,” July 6, 2011, http://www.eda.europa.eu/docs/documents/edrs.pdf, [Last Retrieved December 17, 2013].

[12] Wiegand, M., "EDRS Ongoing Program under Implementation,” EDRS - Globe Net Industry Day Seminar, Munich, Germnay, February 7, 2013.

[13] Nufern, “Fiber Amplifiers, CW Platform, 1.0 Micron”, November 2013, http://www.nufern.com/pam/fiber_amplifiers/, [Last Accessed January 2014].

[14] Ballweg, R. and Wallrap, F., "EDRS Operations at GSOC - Relevant Heritage and New Developments,” SpaceOps 2012 Conference, Stockholm, Sweden, June 2012.

[15] Moll, F. and Knapek, M., "Wavelength Selection Criteria and Link Availability due to Cloud Coverage Statistics and Attenuation Affecting Satellite, Aerial, and Downlink Scenarios,” Proceedings of SPIE, Vol. 6709, 2007, pp. 670916-1 -12.

[16] Churnside, J. and Shaik, K., "Atmospheric Propagation Issues Relevant to Optical Communications,” NOAA Technical Memorandum ERL WPL-159, Boulder, Colorado, 1989.

[17] King, M., Cross, J., Wharton, S., and Myers, M., “EOS Data Products Handbook Volume 1 Revided 2004,”, NASA Goddard Space Flight Center, Greenbelt, Maryland, 2004.

[18] Parkinson, C. and Greenstone, R., “EOS Data Products Handbook Volume 2,”, NASA Goddard Space Flight Center, Greenbelt, Maryland, 2000.

[19] Perlot, N. and Moll, F., "Probability Distribution of Link Durations with N Disruptive Channels: Application to Ground-Space Optical Communications,” Proceedings of SPIE, Vol. 8517, 2012, pp. 851710-1 - 12.

[20] Wood, R. and Field, P., “The Distribution of Cloud Horizontal Sizes,” Journal of Climate, Vol. 24, 2011, pp. 4800-4816. 